\title{
Visualization of utero-ovarian anastomoses: Comparison between patients with and without uterine fibroid
}

\author{
Yasutaka Baba $^{1}$, Sadao Hayashi ${ }^{1}$, Shunichiro Ikeda, Masayuki Nakajo ${ }^{1}$, Mitsushiro Yoshinaga ${ }^{2}$, \\ Tsutomu Douchi ${ }^{2}$ \\ ${ }^{1}$ Department of Radiology, Graduate School of Medical and Dental Sciences, Kagoshima University, Kagoshima, Japan \\ ${ }^{2}$ Department of Obsterics and Gynecology, Graduate School of Medical and Dental Sciences, Kagoshima University, Kagoshima, \\ Japan \\ Email: yasutaka@m3.kufm.kagoshima-u.ac.jp
}

Received 7 October 2012; revised 15 November 2012; accepted 2 December 2012

\begin{abstract}
Purpose: To retrospectively review uteroovarian anastomosis (UOA) visualization during uterine artery embolization (UAE) in patients with or without uterine fibroids (UFs). Material and Methods: 43 patients underwent UAE for uterine fibroids $(n=23)$ and gynecological hemorrhage $(n=20)$. The frequency of angiographic visualization of UOAs was compared between the two groups and analyzed by the Fisher's exact test. Results: Except for one patient with no right uterine artery, 85 uterine arteries were evaluated. Of these 85 uterine arteries, 23 UOA were recognized: 14 UOAs (58\%) (right: 5 [21.7\%], left: 9 [37.5\%]) were visualized in 23 patients with UFs, and 9 UOAs (47.4\%) (right: 4 [21\%], left: 5 [26.3\%]) were visualized in 20 patients with gynecological hemorrhage. In both groups, UOA was observed after UAE in one patient. Statistically significant difference was not observed for UOAs visualized between patients with or without UFs except a group of left UAE in patients with UFs $(P=0.036)$. Conclusion: The frequency of UOAs visualized during angiography was similar between patients with or without UFs. Therefore, UOA should be visualized carefully during UAE in patients with gynecological hemorrhage.
\end{abstract}

Keywords: Uterus; Leiomyoma; Uterine Artery Embolization; Ovary

\section{INTRODUCTION}

Uterine artery embolization (UAE) is now widely accepted as a treatment for uterine fibroids (UFs) with a high success rate and is established as an alternative to hysterectomy or myomectomy [1-3]. However, there are several problems concerning ovarian dysfunction after UAE that have not been overcome [4,5]. A problem with
UAE is utero-ovarian anastomoses (UOAs), which are thought to cause repeated UAE due to technical or ovarian failure [6]. UAE is also performed for genital bleeding. In a series of postpartum hemorrhage, the clinical and hemostasis success rate was high [7]. However, little attention is usually paid to the existence of UOA during UAE for patients without UFs. In addition, UOA should be checked when UAE is performed for genital bleeding in patients without UFs to prevent complications from migrating embolic materials. The purpose of this study is to investigate whether the frequency of visualizing UOA by arteriography is higher in patients with UFs than those without UFs.

\section{MATERIALS AND METHODS}

This was a retrospective study conducted from August 1997 to April 2006. Forty-three patients underwent UAE for UFs $(n=22)$, adenomyosis $(n=1)$, and gynecological hemorrhage $(\mathrm{n}=20)$. All patients (average age, 42 years; range, 17 - 64 years) with UFs and adenomyosis exhibited symptoms of UF and underwent UAE. Patients with gynecological hemorrhage (average age, 42 years; range, 24 - 75 years) included those with cervical cancer (11), uterine sarcoma (1), and postpartum (8).

UAE was performed via the right common femoral artery access. Bilateral uterine arteries were catheterized using a 5-Fr Cobra catheter (Hanako, Cobra type, Tokyo, Japan) under local anesthesia. After performing bilateral uterine arteriography, a 3-Fr microcatheter (Renegade, Boston Scientific, Fremont, CA, USA) was introduced coaxially into the ascending portion of the uterine artery. The gelatin sponge particles used in UAE were made from gelatin sponge sheets (Gelfoam; Upjohn, Kalamazoo, MI, USA) by the surgeons. The sheets were cut and compressed into thin paper-like slices using a scalpel. Then, they were cut into very small fragments using small scissors [8]. In addition to the gelatin sponge, 
microcoils or n-butyl-cyanoacrylate was also used for hemostasis in patients without UFs if necessary.

The frequency of visualizing UOA was compared pre and post UAE between with and without UF patient groups. Fischer exact analysis was performed using a commercially available computer software (MedCalc Software, Mariakerke, Belgium).

\section{RESULTS}

The results are summarized in the Table 1 and Figures 1-4. Except for one patient without a right uterine artery, 85 uterine arteries were evaluated. Of these 85 uterine arteries, 23 UOA were recognized. In patients with UFs, 14 UOA (58\%) (right: 5 [21.7\%], left: 9 [37.5\%]) were visualized. Meanwhile, 9 UOA (47.4\%) (right: 4 [21\%], left: 5 [26.3\%]) were visualized in patients with gynecological hemorrhage. After UAE for UFs, 11/14 UOA (78 \%) (right: 4, left: 7) could not be visualized by pelvic arteriography. After UAE for nonuterine fibroids, 7/9 UOA (78 \%) (right: 3, left 4) could not be visualized by pelvic arteriography. UOA was visualized after UAE in one patient from each group. There was no statistically significant difference in the UOAs visualized or complications between patients with or without UFs (Tables 1). However, there is a statistically significant difference between pre and post left UAE patients with UFs ( $\mathrm{P}=$ 0.036).

Table 1. Visualization of utero-ovarian (UOA) between uterine fibroid (UF) and non-UF.

\begin{tabular}{|c|c|c|c|c|c|c|c|c|}
\hline \multicolumn{9}{|c|}{ UOA } \\
\hline \multirow[t]{4}{*}{ Group } & \multicolumn{4}{|c|}{ Right } & \multicolumn{4}{|c|}{ Left } \\
\hline & \multicolumn{2}{|c|}{ Before UAE } & \multicolumn{2}{|c|}{ After UAE } & \multicolumn{2}{|c|}{ Before UAE ${ }^{*}$} & \multicolumn{2}{|c|}{ After UAE ${ }^{*}$} \\
\hline & + & - & + & - & + & - & + & - \\
\hline & 5 & 18 & 1 & 22 & 9 & 15 & 2 & 22 \\
\hline Non-UF & 4 & 15 & 2 & 17 & 5 & 14 & 1 & 18 \\
\hline
\end{tabular}

*There is a statistically significant difference between before and after left UAE groups in patients with uterine fibroid (UF).

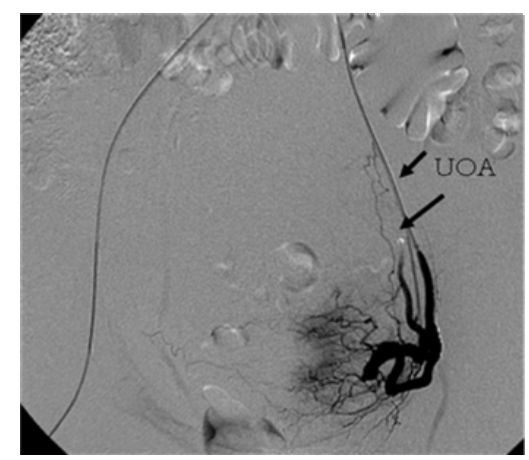

(a)

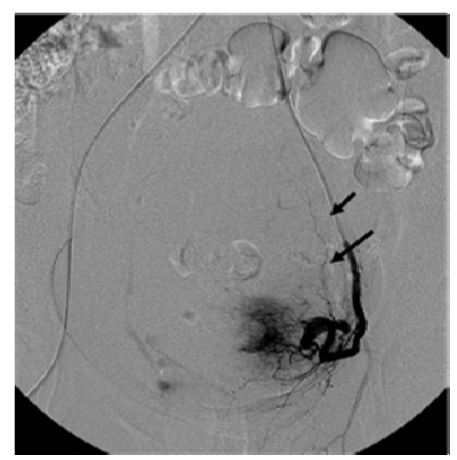

(b)

Figure 1. 35 year-old woman with adenomyosis. (a) Left uterine arteriography before embolization shows uteroovarian anastomses (UOA); (b) Left uterine arteriography after embolization shows persistent visualization of UOA.

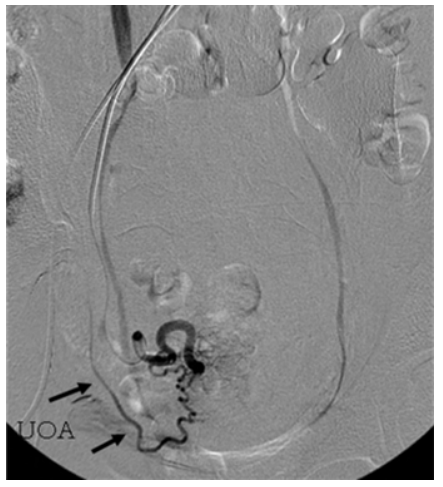

(a)

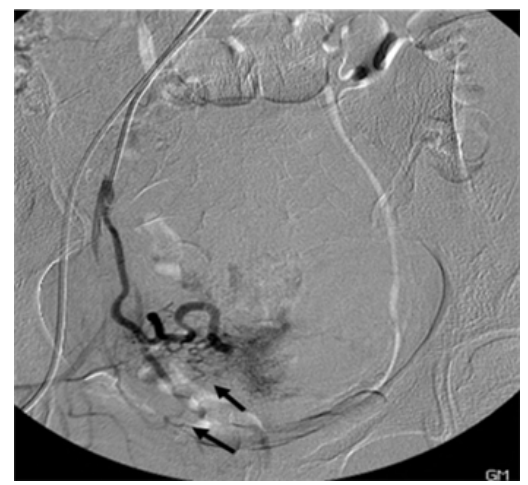

(b)

Figure 2. 40 year-old woman with uterine fibroid. (a) Right uterine arteriography before embolization shows UOA; (b) Right uterine arteriography after embolization shows poorly visualization of UOA. 


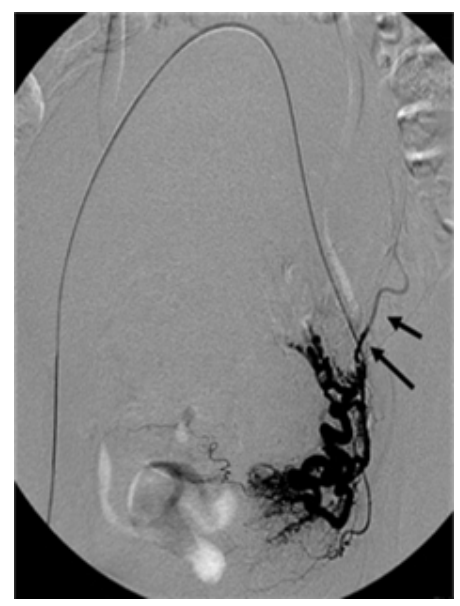

(a)

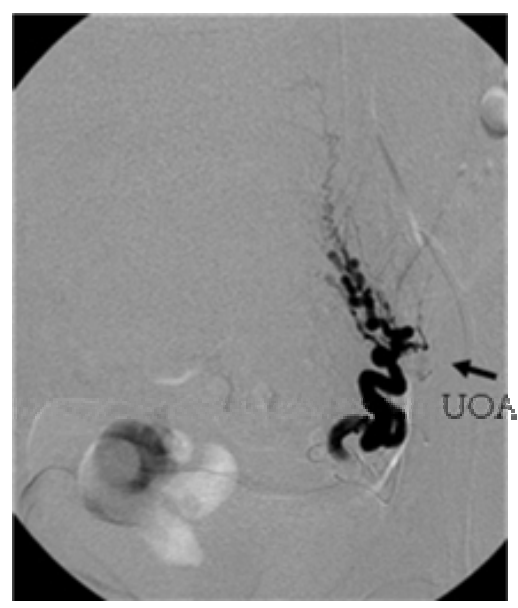

(b)

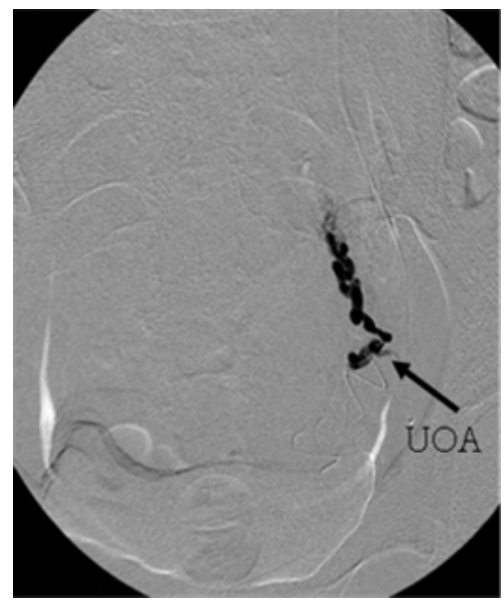

(c)

Figure 3. 49 year-old woman with uterine fibroid; (a) Left; (b) Right: Left uterine arteriography before embolization shows UOA from ascending portion of uterine artery; (c) After tip of the microcatheter is placed at distal site of UOA, UAE is performed to prevent embolizing UOA.

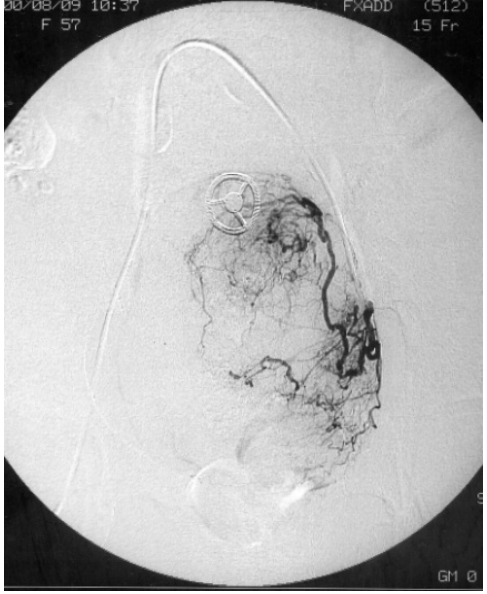

(a)

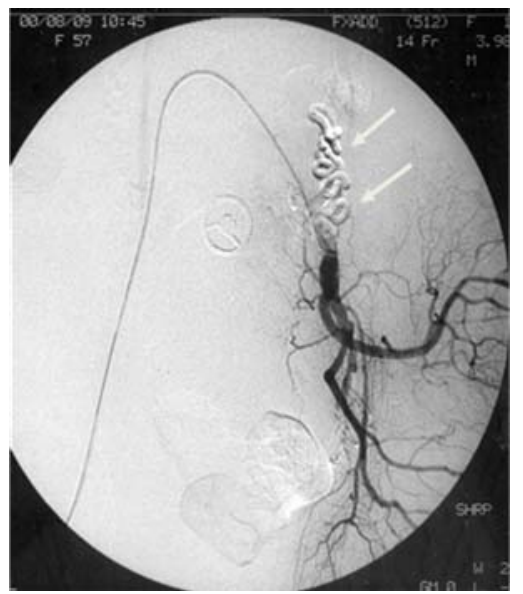

(b)

Figure 4. 45 year-old woman with symptoms of genital bleeding from uterine cancer. (a) Angiogram can not depict the appearance of UOA; (b) However, angiogram immediately after UAE shows the accumulation of embolic materials in the left UOA (arrows).

\section{DISCUSSION}

UOA is considered a cause of technical and ovarian failure after UAE [6]. Previous studies have shown that UOA detected by uterine angiography or aortography is associated with repeated UAE and ovarian dysfunction [1,7-9].

UOA usually exists anatomically, but the frequency of visualizing UOA by arteriography is low [10]. It is hypertrophied in the presence of uterine or ovarian lesions $[9,10]$. In previous studies, UOAs were. Visualized by arteriography in $46.9 \%$ of 32 patients $[9,10]$ with benign ovarian lesions. In our study, ovarian lesions were absent in patients without UFs. Because patients without UFs were represented as a group of nonovarian lesions in our study, there was no correlation with the frequency of UOA visualization between patients with and without UFs or ovarian lesions.

We did not evaluate the relationship between ovarian dysfunction and UOA visualization because of a reported age-related bias in those aged over 45 years and suffering from menopause [5,11]. Kim et al reported 8 patients (38\%) in whom UOA could be visualized angiographically and who underwent hysterectomy with adnexae removal after UAE [12].

Resected specimens showed embolic materials in the adnexa and were histologically viable without evidence of ischemic change or infarction [12]. In both groups in our study, UOA was visualized after UAE. In these patients, we noticed the presence of UOA as the embolic 
materials flowed into UOA [13]. Therefore, we should be careful when a nonvisualized UOA exists during UAE.

In conclusion, the frequency of visualizing UOAs during pelvic arteriography was similar between patients with and without UFs. Therefore, UOA should be visualized carefully during UAE in patients with gynecological hemorrhage.

\section{REFERENCES}

[1] Lohle, P.N., Voogt, M.J., De Vries, J., Smeets, A.J., Vervest, H.A., Lampmann, L.E., et al. (2008) Long-term outcome of uterine artery embolization for symptomatic uterine leiomyomas. Journal of Vascular and Interventional Radiology, 19, 319-326. doi:10.1016/j.jvir.2007.10.011

[2] Walker, W.J. and Barton-Smith, P. (2006) Long-term follow up of uterine artery embolisation-An effective alternative in the treatment of fibroids. BJOG, 113, 464468. doi:10.1111/j.1471-0528.2006.00885.X

[3] Spies, J.B., Cornell, C., Worthington-Kirsch, R., Lipman, J.C. and Benenati, J.F. (2007) Long-term outcome from uterine fibroid embolization with tris-acryl gelatin microspheres: results of a multicenter study. Journal of Vascular and Interventional Radiology, 18, 203-207. doi:10.1016/j.jvir.2006.12.006

[4] Nikolic, B., Spies, J.B., Lundsten, M.J. and Abbara, S. (2000) Patient radiation dose associated with uterine artery embolization. Radiology, 214, 121-125.

[5] Spies, J.B., Roth, A.R., Gonsalves, S.M. and MurphySkrzyniarz, K.M. (2001) Ovarian function after uterine artery embolization for leiomyomata: Assessment with use of serum follicle stimulating hormone assay. Journal of Vascular and Interventional Radiology, 12, 437-442. doi:10.1016/S1051-0443(07)61881-8

[6] Kim, H.S., Paxton, B.E. and Lee, J.M. (2008) Long-term efficacy and safety of uterine artery embolization in young patients with and without uteroovarian anasto- moses. Journal of Vascular and Interventional Radiology, 19, 195-200. doi:10.1016/j.jvir.2007.08.014

[7] Eriksson, L.G., Mulic-Lutvica, A., Jangland, L. and Nyman, R. (2007) Massive postpartum hemorrhage treated with transcatheter arterial embolization: Technical aspects and long-term effects on fertility and menstrual cycle. Acta Radiologica, 48, 635-642. doi:10.1080/02841850701370683

[8] Katsumori, T., Nakajima, K., Mihara, T. and Tokuhiro, M. (2002) Uterine artery embolization using gelatin sponge particles alone for symptomatic uterine fibroids: Midterm results. American Journal of Roentgenology, 178, 135139.

[9] Karlsson, S. and Jonsson, K. (1980) Angiography of the ovarian artery in adnexal lesions. Acta Radiologica Diagnosis, 21, 739-746.

[10] Karlsson, S. and Persson, P.H. (1980) Angiography in uterine and adnexal tumors. Acta Radiologica Diagnosis, 21, 11-20.

[11] Kim, H.S., Tsai, J., Lee, J.M., Vang, R., Griffith, J.G. and Wallach, E.E. (2006) Effects of utero-ovarian anastomoses on basal follicle-stimulating hormone level change after uterine artery embolization with tris-acryl gelatin microspheres. Journal of Vascular and Interventional Radiology, 17, 965-971. doi:10.1097/01.RVI.0000220425.23309.15

[12] Kim, H.S., Thonse, V.R., Judson, K. and Vang, R. (2007) Utero-ovarian anastomosis: Histopathologic correlation after uterine artery embolization with or without ovarian artery embolization. Journal of Vascular and Interventional Radiology, 18, 31-39. doi:10.1016/j.jvir.2006.10.008

[13] Wolanske, K.A., Gordon, R.L., Kerlan Jr., R.K., Wilson, M.W., LaBerge, J.M. and Jacoby, A.F. (2003) Reversal of flow in the ovarian artery during uterine artery embolization. Journal of Vascular and Interventional Radiology, 14, 785-787. doi:10.1097/01.RVI.0000079989.80153.28 